\title{
Effect of collagen fibres and elastic lamellae content on the mechanical behaviour of abdominal aortic aneurysms
}

\author{
MAGDALENA KoBIELARZ* \\ Department of Mechanics, Materials and Biomedical Engineering, Faculty of Mechanical Engineering, \\ Wrocław University of Science and Technology, Wrocław, Poland.
}

\begin{abstract}
Purpose: The main purpose of this study was a detailed analysis of the mechanical and structural characteristics of human abdominal aneurysms in comparison with normal abdominal aortae and determination of the correlations between their mechanical behaviour and the microstructural content. Methods: Various mechanical properties, i.e., mechanical failure properties, elastic moduli, inflection point coordinates, index of anisotropy and incompressibility were determined under uniaxial loading conditions in the circumferential and axial directions. Constitutive parameters were derived from the commonly used constitutive model proposed by Holzapfel et al. [9]. The microstructural arrangement was examined by histological staining supported by scanning electron microscopy analysis. The content of collagen fibres and elastic lamellae was tested in relation to mechanical properties and constitutive parameters. Results: Significant differences were found in the microstructural arrangement and layer composition of the aneurysmal specimens, compared to the normal aorta group. The mechanical properties and constitutive parameters of the aneurysmal specimens were significantly altered, indicating a weakening of the load-bearing properties of the walls of the aneurysms. A comparative analysis discovered significant correlations between structural composition and mechanical parameters, in particular with respect to the number of collagen fibres and failure stress, which can be important for clinical evaluation of abdominal aortic aneurysm (AAA) rupture. Conclusions: Changes in the content of collagen fibres and elastic lamellae correlate with mechanical and constitutive parameters, indicating AAA severity.
\end{abstract}

Key words: abdominal aortic aneurysm, mechanical properties, constitutive modelling, uniaxial tensile loading, collagen fibres, elastic lamellae

\begin{tabular}{|c|c|}
\hline & Abbreviations \\
\hline$\sigma_{M}$ & - mechanical failure stress, tensile strength \\
\hline$M M T$ & - maximum tangent modulus \\
\hline$\lambda_{I P}, \sigma_{I P}$ & $\begin{array}{l}\text { - stretch ratio and stress at inflection points (IP), coor- } \\
\text { dinates of inflection point }\end{array}$ \\
\hline$M T M_{L}$ & $\begin{array}{l}\text { - maximum tangent modulus for the low-stretch do- } \\
\text { mains }\end{array}$ \\
\hline$M T M_{H}$ & $\begin{array}{l}\text { - maximum tangent modulus for the high-stretch } \\
\text { domains }\end{array}$ \\
\hline$A$ & - anisotropy index \\
\hline $\operatorname{det} \boldsymbol{F}$ & - incompressibility \\
\hline $\boldsymbol{F}$ & - deformation gradient tensor \\
\hline$\lambda_{a}, \lambda_{c}, \lambda_{r}$ & $\begin{array}{l}\text { - principal stretches in circumferential, axial and radial } \\
\text { directions }\end{array}$ \\
\hline$\Psi_{v o l}$ & - volumetric term of the strain energy density function \\
\hline & - isotropic term of the strain energy density function \\
\hline
\end{tabular}

$\Psi$

$\boldsymbol{J}$

C - right Cauchy-Green deformation tensor

$I_{1}, I_{4}, I_{6} \quad$ - the first, the fourth and the sixth invariant of the Cauchy-Green deformation tensor

$\boldsymbol{M}-$ vectors of the collagen fibres mean orientation

$\mu, k_{1}, k_{2}, \alpha$ - material parameters derived from the constitutive model fitted to experimental data

$N_{E}, N_{C} \quad-$ elastic lamellae and collagen fibres number

\section{Introduction}

According to the definition by Society for Vascular Surgery (SVS) and International Society for Cardiovascular Surgery (ISCVS) of 1991, an abdominal aor-

* Corresponding author: Magdalena Kobielarz, Department of Mechanics, Materials and Biomedical Engineering, Faculty of Mechanical Engineering, Wrocław University of Science and Technology, ul. Łukasiewicza 7/9, 50-371 Wrocław, Poland. E-mail: magdalena.kobielarz@pwr.edu.pl

Received: April 18th, 2020

Accepted for publication: June 2nd, 2020 
tic aneurysm (AAA) is a permanent localized dilation of a normal abdominal aorta (NAA) by at least $50 \%$, compared to the normal diameter [10]. In a clinical setting, an AAA is diagnosed in the cases where the diameter of an infrarenal aortic aneurysm exceeds $30 \mathrm{~mm}$ [19]. The incidence of AAA is $4-10 \%$ of the global population and the likelihood of formation of this type of aneurysm increases with age, especially after age of 50 [1]. The most dangerous complication of this disease is aneurysm rupture, which is a direct life threat that requires immediate treatment. The overall mortality rate associated with AAA rupture reaches up to $80-90 \%$ [18].

An AAA develops as a result of multifactorial, pathological remodelling of the extracellular matrix of the aortic wall [26], which is built mostly of elastic lamellae and types I and III collagen fibres [12]. Fibrotic components are degraded by proteolytic enzymes from the endopeptidase family, mainly extracellular matrix metalloproteinases [25], [26]. Degradation of elastic lamellae and, consequently, of elastic fibres, is considered the primary cause of aneurysm development, while the destruction of collagen leads to a decrease in tensile strength and is the main cause of aneurysm rupture [25]. From a mechanical point of view, AAA rupture can be considered a classic case of material destruction that occurs as a result of excessive loading on the vessel wall, insufficient material strength, or both of these factors simultaneously [13]. The strength of aneurysmal walls can decrease by as much as $50-60 \%$ compared to normal abdominal aortic walls. In addition, AAA walls are characterised by higher stiffness than normal aortic walls [7], [24], [27], [28].

Both normal and aneurysmal arterial tissues subjected to uniaxial loading exhibit strong nonlinear behaviour with an exponential stiffening effect at high-strain domains that is typical of soft tissues [12], [14]-[17]. Arterial walls are considered anisotropic, incompressible [2], and subjected to finite strains with negligible shear deformation [13], [21]. The passive mechanical behaviour of the arterial tissue is characterized by the strain-energy function $(\Psi)$ of hyperelastic models representing vascular walls as the fibrereinforced composites [9], [13], [21]. It has been proposed that the strain-energy function additively decomposes into an isotropic contribution ( $\left.\Psi_{i s o}\right)$, an anisotropic or at least orthotropic contribution ( $\Psi_{\text {aniso }}$ or $\Psi_{\text {ortho }}$ ), and a contribution of the volumetric part of the deformations $\left(\Psi_{v o l}\right)$ [9]. The isotropic contribution of strain energy represents energy stored in the noncollagenous matrix, i.e., the ground matrix and a portion of randomly distributed fibre reinforcement, and corresponds mainly to the initial stiffness of the arterial wall. The anisotropic term of the strain-energy function refers to energy stored in collagen fibres, i.e., isochoric and anisotropic behaviour of collagen fibres embedded in the extracellular matrix. The anisotropic contribution is connected with stiffness in high-strain domains [6], [12].

In this study, a detailed mechanical characterization of human normal abdominal aortae and AAAs (both from infrarenal aortic part) was performed on a large heterogeneous group of specimens with significant structural diversity. This information can be helpful in defining a model of AAA development based on specific structural changes and corresponding mechanical behaviour and properties. A better understanding of the progression of aneurysm disease requires examination of the correlations between the mechanics and the microstructure, which motivated this study. Therefore, the analysis covered the stressstretch relationships under uniaxial loading conditions and constitutive parameters derived from the commonly used constitutive model proposed by Holzapfel et al. [9] and further microstructural arrangement, obtained by investigation of multiple histological parameters and supported by scanning electron microscopy (SEM) imaging.

\section{Materials and methods}

\subsection{Tissue preparation}

Preparations of AAA walls $(n=96)$ were collected intraoperatively at the Provincial Specialist Hospital in Wrocław, Poland. During elective open repairs of aneurysms, excess tissue was collected from the anterior walls of AAAs, remaining after sealing of the vascular prosthesis. The walls of normal abdominal aortae from the infrarenal section $(n=67)$ were collected post-mortem during forensic medical examination at the Department of Forensic Medicine of Wrocław Medical University within 24 hours of the donors' death. All collected tissues (specimens) were stored in normal saline at $4{ }^{\circ} \mathrm{C}$ until testing, but no longer than 24 hours. Donor information is summarised in Table 1. There were no statistically significant differences in age structure between the AAA and NAA groups. The sex distribution of the donors was similar in both groups. Examinations of tissue material collected from human subjects was approved by the Bioethics Committee of Wrocław Medical University (decision 
No. 374/2009). The research was carried out in accordance with the principles outlined in the Declaration of Helsinki.

Table 1. Summary of donors information

\begin{tabular}{|l|c|c|c|}
\cline { 2 - 4 } \multicolumn{1}{c|}{} & NAA & AAA & $\begin{array}{c}p \text {-values/ } \\
\text { statistical test }\end{array}$ \\
\hline Age & $66 \pm 11$ & $68 \pm 9$ & $p=0.183$ \\
[years] & $53-81$ & $58-77$ & Student's $t$-test \\
\hline Sex & $\mathrm{M}=57(85.1 \%)$ & $\mathrm{M}=87(90.6 \%)$ & $p=0.109$ \\
{$[\mathrm{M} / \mathrm{F}]$} & $\mathrm{F}=10(14.9 \%)$ & $\mathrm{F}=9(9.4 \%)$ & Chi-square test \\
\hline Diameter & $20.6 \pm 0.9$ & $79.4 \pm 18.8$ & $p=0.0009$ \\
{$[\mathrm{~mm}]$} & $17-28$ & $53-107$ & Student's $t$-test \\
\hline
\end{tabular}

Specimens of AAAs and NAAs were excised for mechanical tests and structural analyses. The specimens for testing mechanical properties were punched out with a punch tool, with a minimum of two specimens for each two mutually perpendicular directions: circumferential (marked as symbol "c" and specimens were identified as AAAc and NAAc) and axial (marked as symbol "a" and specimens were identified as AAAa and NAAa) (Fig. 1), and then were placed in normal saline cooled to $4{ }^{\circ} \mathrm{C}$. From the remaining tissue, two specimens - one for histological and second for ultrastructural examinations were excised with a scalpel, approx. $10 \mathrm{~mm}^{2}$ in size. The specimen for histological examination was placed in $4 \%$ formalin aqueous solution $(\mathrm{pH}=7.4)$ and the specimen for ultrastructural examination was placed in $2.5 \%$ glutaraldehyde solution on phosphate buffer.

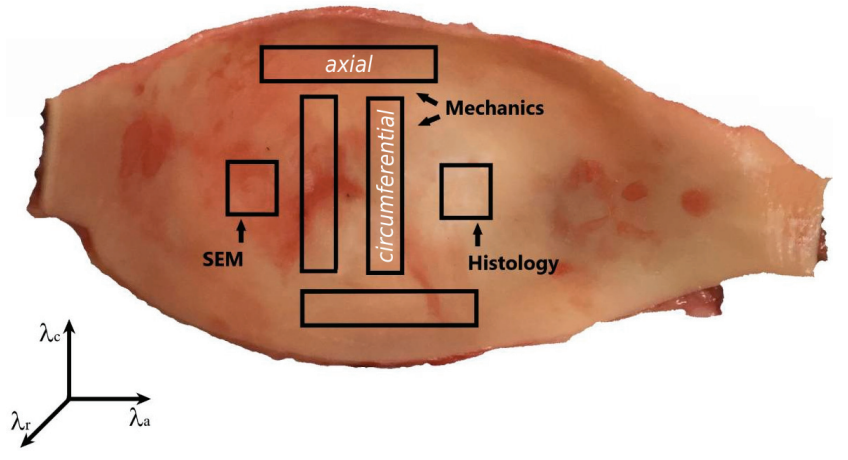

Fig. 1. Representative fragment of the abdominal aortic aneurysm (AAA) wall; contours mark the specimens excised for the respective examinations

\subsection{Structural investigation}

\section{Histology}

Arterial specimens fixed in $4 \%$ formaldehyde solution were embedded in paraffin and sectioned at $5 \mu \mathrm{m}$.
Three types of histological stains were used, namely, standard staining with haematoxylin and eosin (H \& E) and two specific, mutually complementary staining techniques: elastic van Gieson's and Verhoeff's elastic stains. H \& E staining was used to visualize cellularity, calcification content, and general tissue architecture. Elastic van Gieson's and Verhoeff's elastic stains allow to distinguish between elastic lamellae and collagen fibres, hence they were performed to visualize fibrotic composition. Preparations obtained by staining with the specific stains were used to perform histometric analyses using ImageJ software (ver. 1.50b). Elastic lamellae (stained black) and collagen fibres (stained red) was counted. Other connective tissue components and smooth muscle cells stained yellow to brown were omitted. A total of 10 through-thickness lines were drawn perpendicular to the sections, and the elastic lamellae and collagen bundles crossing each of the lines were counted and averaged for individual specimens. In addition, calculations were made of the total wall thickness of individual vessels, layer thickness, and relative thickness of the individual layers as a percentage of the whole wall (intima: media:adventitia ratio, indexed as I:M:A ratio) [22]. Analyses were carried out using a light microscope (AxioImager M1m, Zeiss).

\section{Scanning electron microscopy}

Ultrastructural analysis was performed using a SEM microscope (Phenom ProX, Thermo Fisher Scientific). The fixed material was sliced on a cryostat (Hyrax C50, Zeiss) into $15 \mu \mathrm{m}$-thick sections and attached to specimen stubs with a carbon tape.

\subsection{Mechanical testing}

The initial geometric dimensions of the specimens were as follows: length $l_{0}=25( \pm 0.5) \mathrm{mm}$ and width $w_{0}=5( \pm 0.1) \mathrm{mm}$. The width-to-length (WL) ratio was maintained at $0.2: 1$ as the most suitable for tensile testing [14], [29]. The specimens were mounted on a testing machine (Synergie 100, MTS) with flat clamps covered with rigid elastomer. The specimens were initially pre-loaded to $0.01 \mathrm{~N}$. They were then prestretched for five loading-unloading cycles to $10 \%$ strain. The pre-stretched specimens were quasi-statically stretched to failure at a constant rate of $2 \mathrm{~mm} / \mathrm{min}$. The testing was conducted at room temperature (22 $\pm 1{ }^{\circ} \mathrm{C}$ ) and the specimens were moistened by dripping on them a $0.9 \%$ saline solution.

During tensile testing of the specimens, their geometric dimensions, including length $(l)$, width $(w)$, and 
(a)

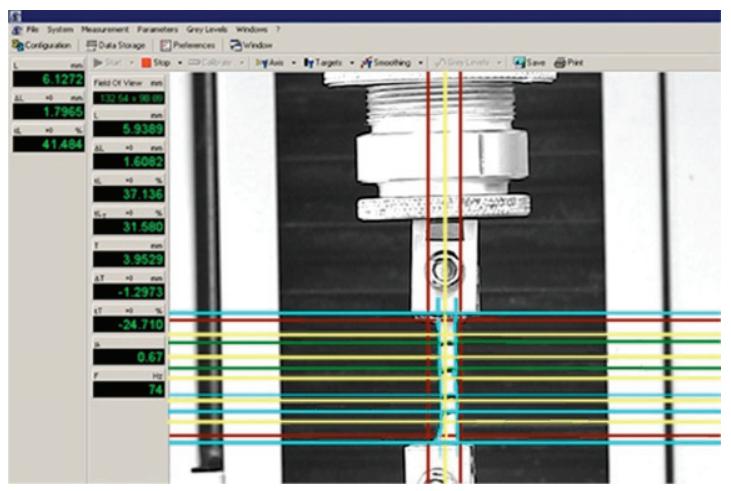

(b)

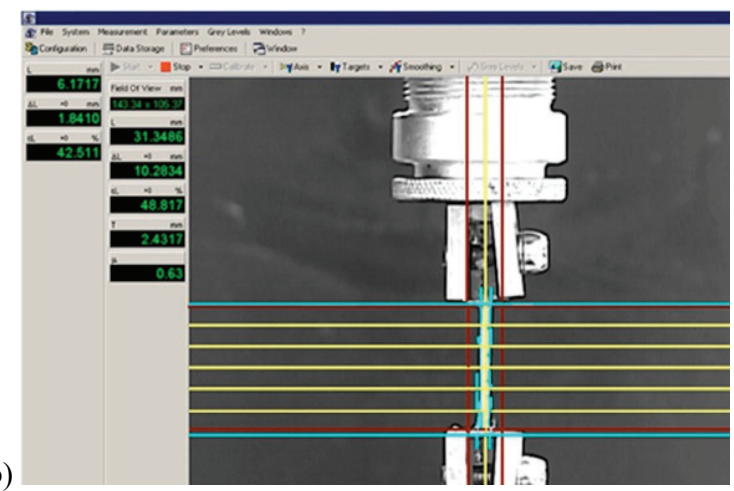

Fig. 2. Visualisation of the process of recording the geometric dimensions of the specimens:

(a) in the frontal plane, and (b) in the lateral plane

thickness $(t)$, were measured using a video extensometer (ME 46-350, Messphysik). The lengths of the specimens were determined in both frontal and lateral planes by measuring the distances between contrasting markers, which were placed on the surfaces of the specimens. In the frontal plane, six markers were placed (spaced $5 \mathrm{~mm}$ apart), which divided the specimens into five measurement sections. In the lateral plane, on the other hand, two markers were placed at the ends of each specimen, right next to the edges of the mounting clamps. Width and thickness were measured in five measurement sections based on the analysis of specimen contours, which contrasted with the white background (Fig. 2).

Based on uniaxial tensile tests, the Cauchy stressstretch ratio relationships were determined for all the specimens. The mechanical parameters were obtained from the plots in the following steps (Fig. 3) discussed in previously published papers [6], [8], [13], [14], [31]. Firstly, at the maximum point of the curve, the stress $\left(\sigma_{M}\right)$ values were determined, representing the mechanical failure of the specimens under uniaxial loading, and the maximum tangent modulus (MTM), determined as the maximum tangential slope to the Cauchy stress-stretch ratio curves. Secondly, inflection points (IPs) were determined as the maximum curvature based on a derivative and defined as the maximum change of slope. The IP coordinates $\left(\lambda_{I P}, \sigma_{I P}\right)$ were also determined. Then, elastic moduli for the lowstretch $\left(M T M_{L}\right)$ and high-stretch $\left(M T M_{H}\right)$ domains divided by IPs were defined based on the linear fitting of the curve separately for low- and high-stretch domains. All three elastic moduli $\left(M T M, M T M_{L}, M T M_{H}\right)$ represented material properties related to stiffness. Finally, the anisotropy $(A)$ and incompressibility $(\operatorname{det} \boldsymbol{F})$ indices of NAAs and AAAs were assessed on the basis of the principal stretches associated with the radial $\left(\lambda_{r}\right)$, circumferential $\left(\lambda_{c}\right)$, and axial $\left(\lambda_{a}\right)$ directions determined by the video extensometer. The anisotropy index and incompressibility were calculated based on the relationship between the circumferential and axial stretches at the IPs as follows: (a)

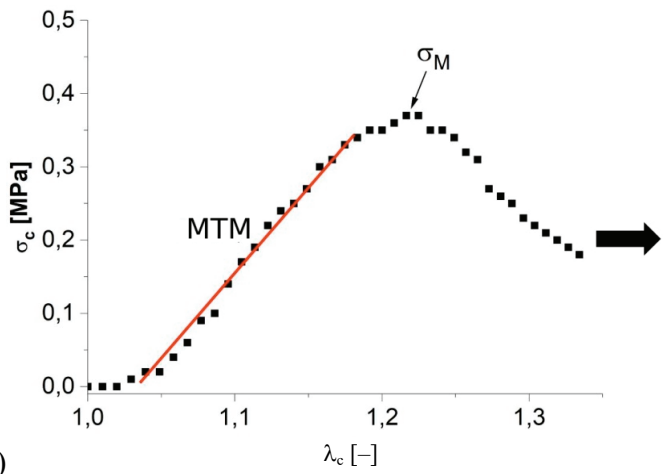

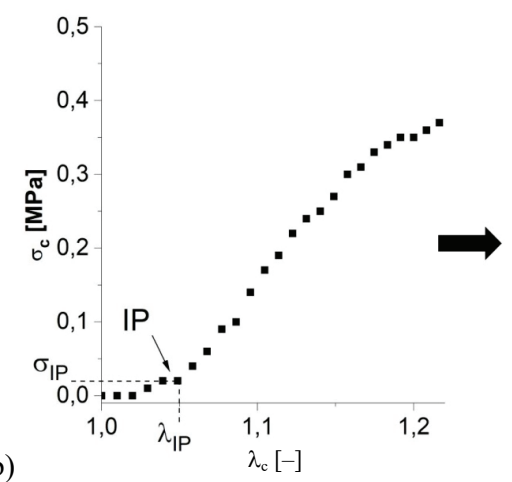

(b)

(c)

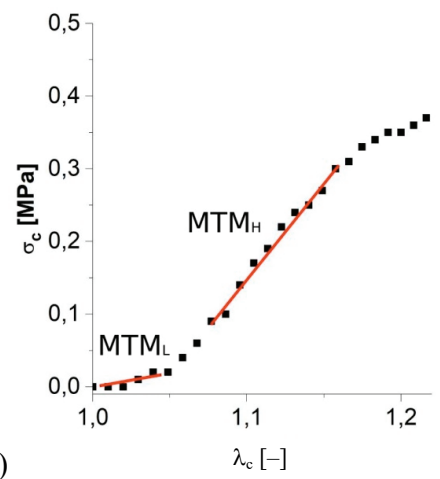

Fig. 3. Pathway of determination of the mechanical properties: a) maximum stress $\left(\sigma_{M}\right)$ and maximum tangent modulus (MTM); b) inflection point (IP) coordinates $\left(\lambda_{I P}, \sigma_{I P}\right)$; and c) maximum tangent modulus for the low-stretch $\left(M T M_{L}\right)$ and high-stretch $\left(M T M_{H}\right)$ domains 


$$
A=\frac{\lambda_{a}-\lambda_{c}}{0.5\left(\lambda_{a}+\lambda_{c}\right)}
$$

The anisotropy index becomes negative if the tissue is more compliant circumferentially than axially. The incompressibility of the material is preserved when the product of the stretches is equal to unity, assuming there is no shear deformation:

$$
\operatorname{det} \boldsymbol{F}=\lambda_{a} \lambda_{c} \lambda_{r},
$$

where $\boldsymbol{F}$ is the deformation gradient tensor, and $\lambda_{a}, \lambda_{c}$, and $\lambda_{r}$ are the principal stretches in the circumferential, axial, and radial directions, respectively.

\subsection{Material model}

The hyperelastic anisotropic model for incompressible materials, proposed by Holzapfel et al. in 2000 [9], was utilized to describe the mechanical behaviour of arterial tissues:

$$
\Psi=\Psi_{v o l}+\Psi_{i s o}+\Psi_{\text {aniso }} .
$$

The first term of the strain energy density, $\Psi_{v o l}=$ $\Psi_{v o l}(J)$, represents volume changes and is dependent on the purely volumetric part of the deformation expressed by the Jacobian of the deformation gradient, defined as $J=\operatorname{det} \boldsymbol{F}$.

The second term, $\Psi_{i s o}=\Psi_{i s o}(\boldsymbol{C})$, represents the isochoric behaviour of the isotropic components of the aortic wall and is dependent on the right CauchyGreen deformation tensor $\boldsymbol{C}$, defined as $\boldsymbol{C}=\boldsymbol{F}^{T} \boldsymbol{F}$ (where $\boldsymbol{F}$ is the deformation gradient tensor), and is expressed by the neo-Hookean function:

$$
\Psi_{i s o}(\boldsymbol{C})=\frac{\mu}{2}\left(I_{1}-3\right),
$$

where $\mu$ is a stress-like material parameter describing the stiffness of the matrix and $I_{1}$ is the first invariant of the Cauchy-Green deformation tensor $\left(I_{1}=\operatorname{tr} \boldsymbol{C}\right)$.

The last term, $\Psi_{\text {aniso }}=\Psi_{\text {aniso }}(\boldsymbol{C}, \boldsymbol{M})$, is the anisotropic part of the energy function denoting the contribution of two symmetrical and mechanically equivalent families of collagen fibres reinforcing the vessel wall. $\Psi_{\text {aniso }}$ depends on the right Cauchy-Green deformation tensor $\boldsymbol{C}$, and vectors $\boldsymbol{M}$ describing the mean orientation of collagen fibres in the reference configuration, and is expressed as:

$$
\Psi_{\text {aniso }}(\boldsymbol{C}, \boldsymbol{M})=\frac{k_{1}}{2 k_{2}} \Sigma_{i=4,6}\left(e^{k_{2}\left(I_{i}-1\right)^{2}}-1\right),
$$

where $k_{1}>0$ is a stress-like parameter related to the stiffness of the fibres, $k_{2}>0$ is a dimensionless mate- rial parameter related to the degree of non-linearity resulting from the progressive nature of fibre behaviour, and $I_{4} \geq 1$ and $I_{6} \geq 1$ are the invariants related to the mechanical response of the fibres in the preferential directions of $\boldsymbol{M}_{4}$ and $\boldsymbol{M}_{6}$, respectively, expressed as:

$$
I_{i}=\boldsymbol{C}: \boldsymbol{M}_{i} \otimes \boldsymbol{M}_{i}, i=4,6 .
$$

The physical meaning of $I_{4}$ and $I_{6}$ is the square of the fibres stretches defined in the reference configuration. Assuming that no shear loads occur under loading conditions and no components are present in the radial direction, the expression of the invariants $I_{4}$ and $I_{6}$ becomes simply:

$$
I_{4}=I_{6}=\lambda_{c}^{2} \cos ^{2} \alpha+\lambda_{a}^{2} \sin ^{2} \alpha,
$$

where $\alpha$ is the angle between the circumferential direction and the main directions of the fibres.

Hence, the material model chosen as a constitutive relation to describe the mechanical behaviour of the aortic walls contained four parameters, of which three were material i.e., $\mu, k_{1}$, and $k_{2}$, and one is structural, i.e., $\alpha$. However, the structural orientation of the fibres within individual specimens was not investigated, hence $\alpha$ was treated here as a phenomenological parameter and was determined by fitting the model to the data. The constitutive parameters were determined using a nonlinear least-squares $l$ sgnonlin function in the Matlab software (ver. 2017b). The standard nonlinear Levenberg-Marquardt algorithm was used during the curve fitting process, resulting in a minimization of the objective function expressed as:

$$
\chi^{2}=\sum_{i=1}^{n}\left(\sigma_{c}^{e}-\sigma_{c}^{m}\right)_{i}^{2}+\left(\sigma_{a}^{e}-\sigma_{a}^{m}\right)_{i}^{2},
$$

where $n$ is the number of data points, $\sigma_{c}^{e}$ and $\sigma_{a}^{e}$ are the experimental Cauchy stresses in each $i$-th point of data, and $\sigma_{c}^{m}$ and $\sigma_{a}^{m}$ are the modelled Cauchy stresses predicted by the strain-energy function corresponding with particular data points for both circumferential and axial directions.

The goodness of fit was evaluated by the coefficient of determination $\left(R^{2}\right)$.

\subsection{Statistical analysis}

Statistical analysis was performed using Statistica software (Statistica 13.1, StatSoft) with a statistical significance level of 0.05 . Statistical significance was tested between normal and aneurysmal aortic speci- 
mens using Student's $t$-test for independent groups. The results were presented in the form of means with standard deviations $(\bar{X} \pm \mathrm{SD})$. The correlation between structural and mechanical parameters was analysed using Pearson's correlation coefficient $(R)$ test.

\section{Results}

\subsection{Structural investigations}

Histological images of abdominal aortic walls showed correctly shaped layers (Fig. 4a). The bounda- ries of the layers were clearly marked, which allowed for accurate measurement of their thickness (Table 2). In contrast, histological images of the AAA wall preparations showed degradation of all the layers, especially the tunica intima, and various degrees of blurring of the boundaries between the layers (Figs. 4b, 4c). In the cases where the boundaries of individual layers were distinguishable, their thickness was measured (Table 2).

The tunica media of the NAA walls showed numerous elastic lamellae (E). Collagen fibres (C) observed both in the tunica media and tunica adventitia followed a morphologically normal, wavy course (Fig. 5a). It was noted that the courses of collagen fibres and elastic lamellae were mutually complementary in the

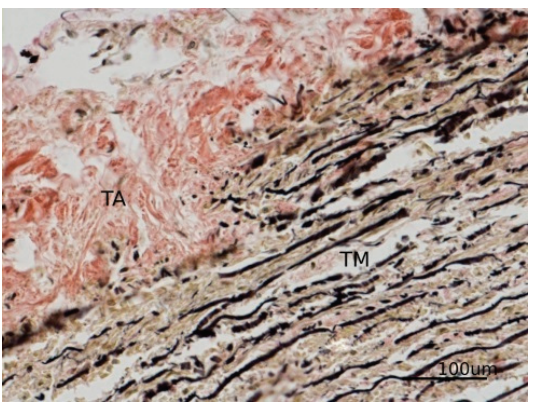

(a)

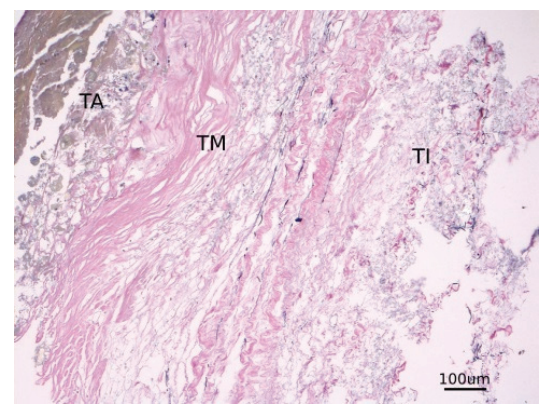

(b)

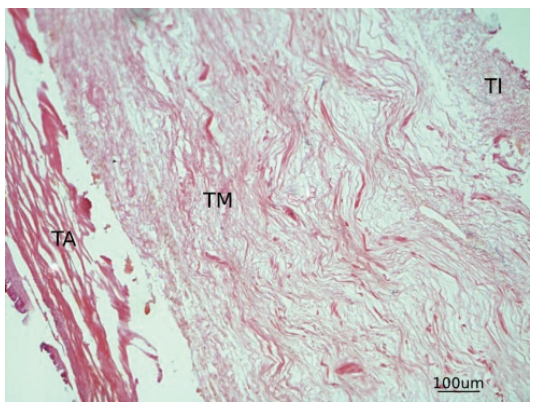

(c)

Fig. 4. Histological images of: (a) walls of a normal abdominal aorta (NAA) with clearly marked elastic lamellae (black bands) stained with Verhoeff's stain, and (b) and (c) AAA walls with layers differentiated with van Gieson's stain. Legend: TA - tunica adventitia, TM - tunica media, TI - tunica intima

Table 2. Thickness of the walls and individual layers of NAAs and AAAs

\begin{tabular}{|c|c|c|c|c|c|c|c|}
\hline & \multicolumn{3}{|c|}{ NAA } & \multicolumn{3}{|c|}{ AAA } & \multirow{2}{*}{$p$-value } \\
\hline & $\bar{X} \pm \mathrm{SD}[\mu \mathrm{m}]$ & Range $[\mu \mathrm{m}]$ & I:M:A [\%] & $\bar{X} \pm \mathrm{SD}[\mu \mathrm{m}]$ & Range $[\mu \mathrm{m}]$ & I:M:A [\%] & \\
\hline $\begin{array}{l}\text { Intima } \\
\text { (I) }\end{array}$ & $272.5 \pm 180.7$ & $51.2 \div 394.9$ & $\begin{array}{c}21 \\
(6 \div 25)\end{array}$ & $63.5 \pm 23.0$ & $43.7 \div 88.7$ & $\begin{array}{c}6 \\
(6 \div 7) \\
\end{array}$ & 0.001 \\
\hline $\begin{array}{l}\text { Media } \\
\text { (M) }\end{array}$ & $702.2 \pm 346.6$ & $427.7 \div 1330.8$ & $\begin{array}{c}54 \\
(47 \div 85)\end{array}$ & $622.4 \pm 201.8$ & $301.6 \div 958.7$ & $\begin{array}{c}65 \\
(45 \div 80)\end{array}$ & 0.037 \\
\hline $\begin{array}{l}\text { Adventitia } \\
\text { (A) }\end{array}$ & $201.7 \pm 105.5$ & $100.5 \div 383.7$ & $\begin{array}{c}15 \\
(11 \div 24)\end{array}$ & $194.1 \pm 52.3$ & $120.0 \div 273.4$ & $\begin{array}{c}20 \\
(18 \div 23)\end{array}$ & 0.074 \\
\hline Wall & $1296.6 \pm 303.9$ & $913.4 \div 1555.7$ & - & $952.9 \pm 233.8$ & $663.0 \div 1187.7$ & - & 0.015 \\
\hline
\end{tabular}
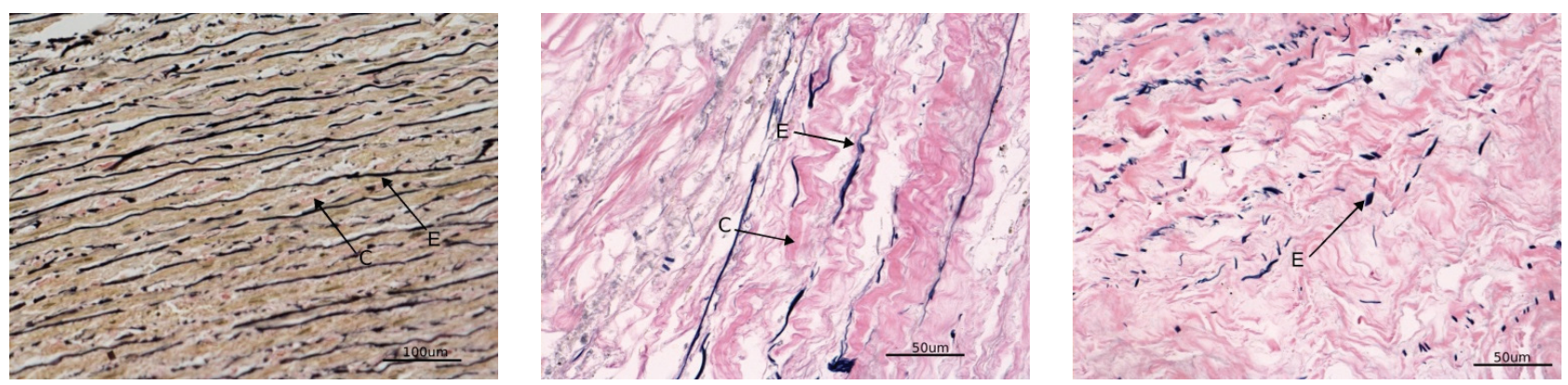

Fig. 5. Histological images of elastic lamellae (E) and collagen fibres (C) showing (a) the tunica media of the abdominal aortic wall (Verhoeff's stain), (b) individual elastic lamellae (E), and (c) fragmentation of elastic lamellae (E) in the AAAs walls (van Gieson's stain) 
(a)

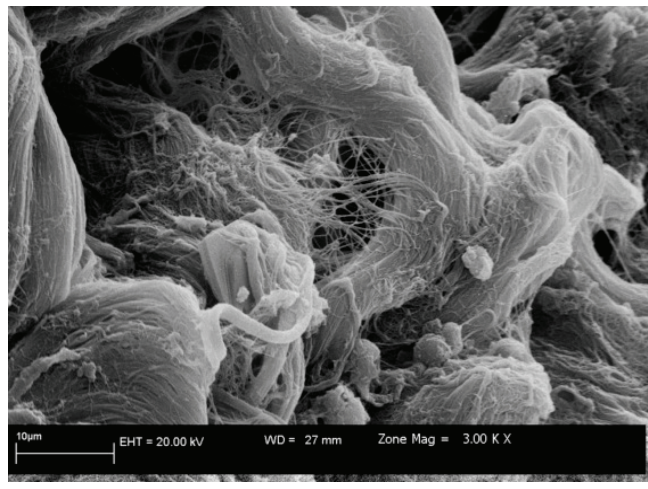

(b)

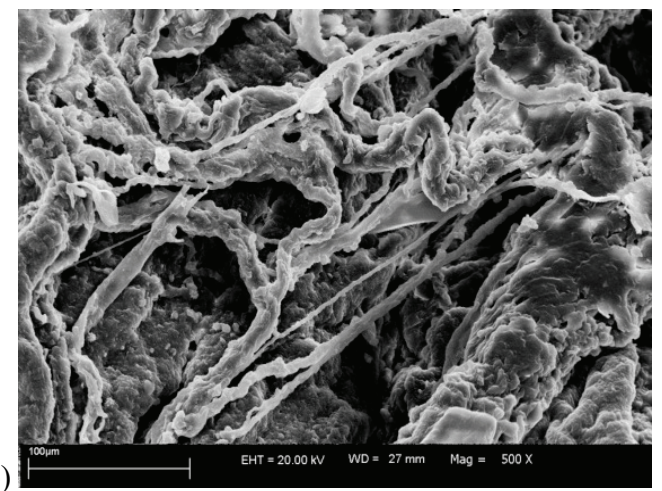

Fig. 6. Scanning electron microscopy images showing elastic lamellae and collagen fibres in the tunica media of (a) the NAA wall, and (b) the AAA wall

wall of a normal vessel. A SEM image also showed characteristic, morphologically normal fibre arrangement (Fig. 6a). Degradation of elastic lamellae was noted for all AAA preparations (Fig. 5b and c). This phenomenon had different intensity in individual cases. In most preparations, the number of fibres was significantly reduced, sometimes to single strands, which were randomly present in the entire histological image (Fig. 5b). Fragmentation of elastic lamellae was also observed (Fig. 5c). The arrangement of collagen fibres in the media and adventitia of AAAs was disturbed. The fibres were often straight (Fig. 5b). This phenomenon was also observed in electron microscope images (Fig. 6b).

Table 3. The number of elastic lamellae $\left(N_{E}\right)$ and collagen fibres $\left(N_{C}\right)$ in NAA and AAA preparations

\begin{tabular}{|l|c|c|c|c|c|}
\cline { 2 - 5 } \multicolumn{1}{c|}{} & \multicolumn{2}{c|}{ NAA } & \multicolumn{2}{c|}{ AAA } & \multirow{2}{*}{$p$-value } \\
\cline { 2 - 5 } \multicolumn{1}{c|}{} & $\bar{X} \pm \mathrm{SD}$ & Range & $\bar{X} \pm \mathrm{SD}$ & Range & \\
\hline$N_{E}$ & $54.24 \pm 9.89$ & $42 \div 72$ & $9.12 \pm 3.24$ & $4 \div 17$ & 0.0001 \\
\hline$N_{C}$ & $83.76 \pm 10.79$ & $64 \div 96$ & $65.84 \pm 18.35$ & $34 \div 88$ & 0.006 \\
\hline
\end{tabular}

NAA

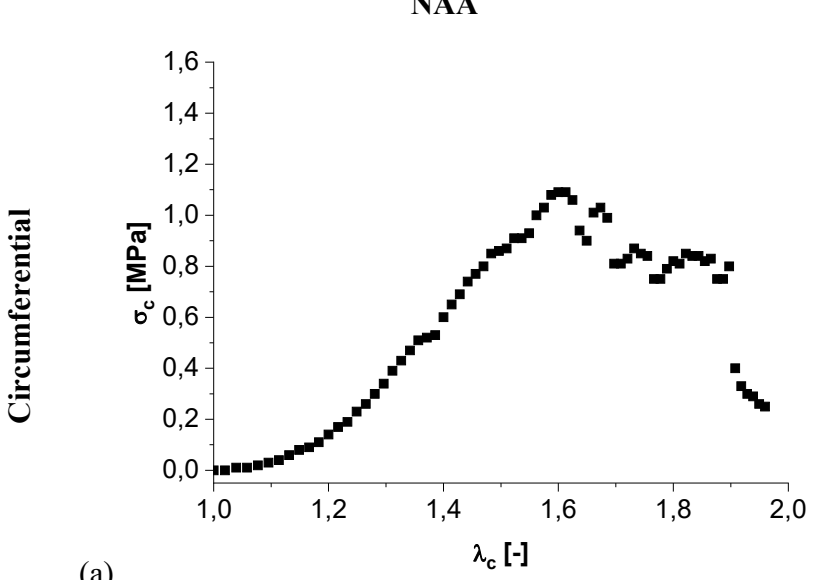

(a)

(b)

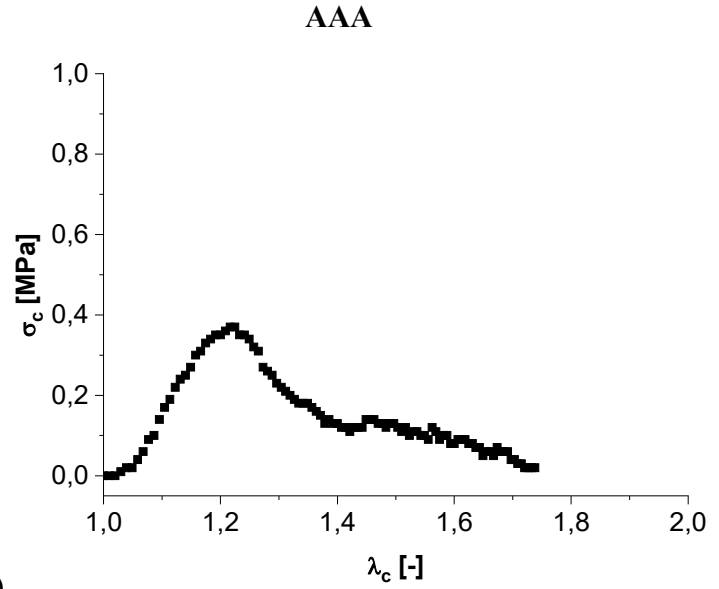

\subsection{Mechanical properties}

The Cauchy stress-stretch ratio curves are highly nonlinear for all tissues (Fig. 7).

The mechanical parameters of aneurysmal and normal aortic walls (Table 4) were significantly different, which was particularly evident in the case of specimens tested in the circumferential direction. In the axial direction, only the $M T M$ was significantly different (higher) for AAA walls compared to NAA walls. In the circumferential direction, aneurysmal walls were characterized by much higher MTM (by approx. 40\%) and much lower strength $\left(\sigma_{M}\right)$ (by approx. $60 \%$ ). In the low-stretch domains, elastic modulus $\left(M T M_{L}\right)$ of AAA walls was significantly lower, and in the range of high-stretch domains $\left(M T M_{H}\right)$ it was significantly higher.

The determinant of the deformation gradient tensor both for AAA and NAA walls was approximately equal to one (Table 5). This means that AAA and NAA walls were incompressible materials. The standard de- 
NAA

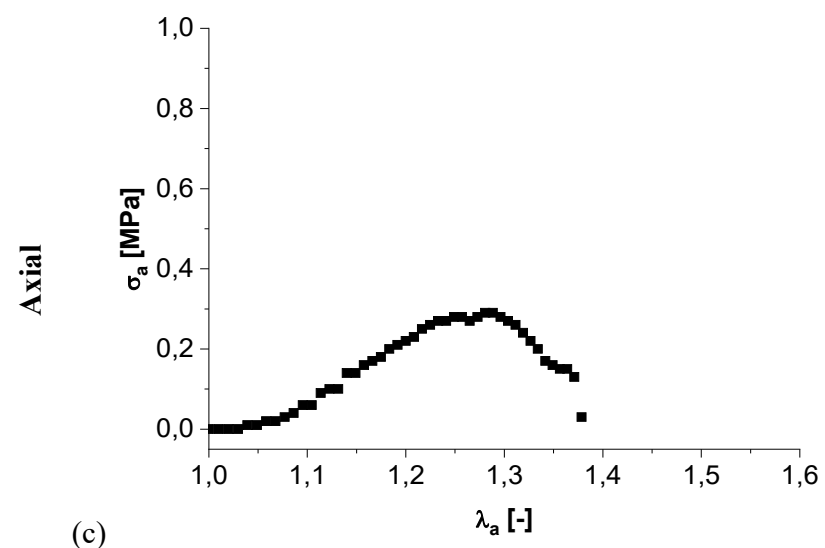

AAA

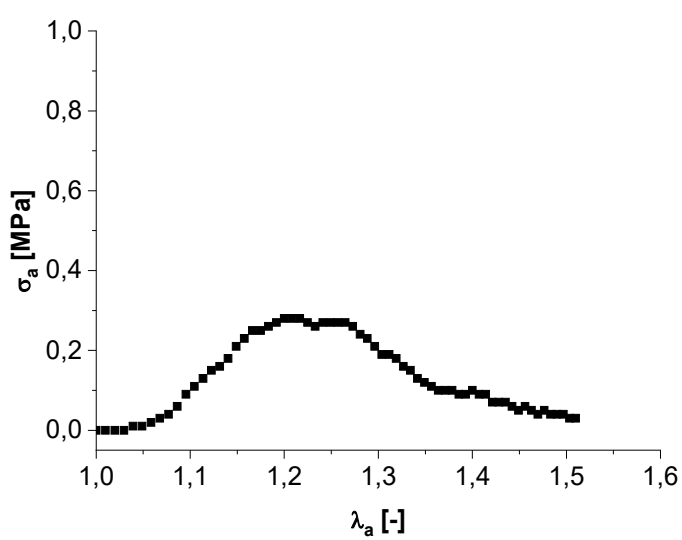

(d)

Fig. 7. Examples of the Cauchy stress-stretch ratio for:

(a) NAAc, (b) AAAc, (c) NAAa, and (d) AAAa

Table 4. Mechanical properties of NAA and AAA walls determined in the circumferential and axial directions based on the Cauchy stress-stretch ratio

\begin{tabular}{|l|c|c|c|c|c|c|}
\cline { 2 - 7 } \multicolumn{1}{c|}{} & \multicolumn{3}{c|}{ Circumferential direction } & \multicolumn{3}{c|}{ Axial direction } \\
\cline { 2 - 7 } \multicolumn{1}{c|}{} & NAAc & AAAc & $p$-value & NAAa & AAAa & $p$-value \\
\hline$\sigma_{M}[\mathrm{MPa}]$ & $1.26 \pm 0.64$ & $0.51 \pm 0.44$ & 0.000001 & $0.49 \pm 0.27$ & $0.37 \pm 0.26$ & 0.081 \\
\hline$M T M[\mathrm{MPa}]$ & $1.01 \pm 0.54$ & $1.72 \pm 1.23$ & 0.007 & $0.83 \pm 0.46$ & $1.27 \pm 1.06$ & 0.048 \\
\hline$\lambda_{I P}[-]$ & $1.19 \pm 0.15$ & $1.06 \pm 0.04$ & 0.004 & $1.08 \pm 0.06$ & $1.06 \pm 0.05$ & 0.097 \\
\hline$\sigma_{I P}[\mathrm{MPa}]$ & $0.12 \pm 0.04$ & $0.04 \pm 0.03$ & 0.002 & $0.03 \pm 0.01$ & $0.02 \pm 0.02$ & 0.169 \\
\hline$M T M_{L}[\mathrm{MPa}]$ & $0.28 \pm 0.17$ & $0.19 \pm 0.16$ & 0.082 & $0.22 \pm 0.09$ & $0.17 \pm 0.11$ & 0.176 \\
\hline$M T M_{H}[\mathrm{MPa}]$ & $2.60 \pm 0.93$ & $3.73 \pm 2.82$ & 0.032 & $1.74 \pm 1.11$ & $2.10 \pm 1.93$ & 0.268 \\
\hline
\end{tabular}

Legend: $M T M-$ maximum tangent modulus, $\sigma_{M}$ - tensile strength, $\lambda_{I P}, \sigma_{I P}-$ coordinates of inflection point, $M T M_{L}$ - maximum tangent modulus for the low-stretch domains, $M T M_{H}$ - maximum tangent modulus for the high-stretch domains.

Table 5. The anisotropy index (A) and incompressibility (det $\boldsymbol{F})$ determined for NAA and AAA walls in the circumferential and axial directions

\begin{tabular}{|c|c|c|c|}
\cline { 2 - 4 } \multicolumn{1}{c|}{} & NAA & AAA & $p$-value \\
\hline$A[-]$ & $-0.093 \pm 0.041$ & $-0.002 \pm 0.001$ & 0.00007 \\
\hline $\operatorname{det} \boldsymbol{F}[-]$ & $0.98 \pm 0.08$ & $0.98 \pm 0.21$ & 0.16 \\
\hline
\end{tabular}

viation for aneurysmal walls was very large $( \pm 0.21)$. It was noted that the presence of inclusions in the aneurysmal wall in the form of intramural thrombi (results not presented here) lowered the value $\operatorname{det} \boldsymbol{F}$. The anisotropy index was clearly different for AAAs and NAAs (Table 5). Anisotropy of the mechanical properties for NAA walls was pronounced and the aortae were more compliant circumferentially than axially. For aneurysmal walls, anisotropy disappeared and AAAs were comparably compliant in the circumferential and axial directions.

\subsection{Material model}

The behaviour of the walls of AAAs and NAAs under mechanical loading conditions was described using the model proposed by Holzapfel et al. [9]. It was noted that three out of four parameters of the model $\left(\mu, k_{1}\right.$, and $\left.k_{2}\right)$ differed significantly between NAAs and AAAs (Table 6). The angle between the circumferential direction and the main directions of the

Table 6. The constitutive parameters for NAA and AAA

\begin{tabular}{|l|c|c|c|}
\cline { 2 - 4 } \multicolumn{1}{c|}{} & NAA & AAA & $p$-value \\
\hline$\mu[\mathrm{MPa}]$ & $0.22 \pm 0.06$ & $0.11 \pm 0.03$ & 0.007 \\
\hline$k_{1}[\mathrm{MPa}]$ & $2.29 \pm 2.03$ & $130.95 \pm 63.05$ & 0.000003 \\
\hline$k_{2}[-]$ & $209.24 \pm 32.61$ & $21.78 \pm 17.35$ & 0.000001 \\
\hline$\alpha\left[^{\circ}\right]$ & $46.89 \pm 1.85$ & $43.72 \pm 25.91$ & 0.14 \\
\hline$R^{2} \min -\max$ & $0.94-0.99$ & $0.93-0.96$ & - \\
\hline
\end{tabular}


fibres $(\alpha)$ was comparable for NAAs and AAAs. It should be emphasized that this parameter was determined based on a fitting procedure, hence it was a phenomenological component of the model.

\subsection{Assessment of the impact of structural changes on the mechanical properties of the AAA walls}

Based on the tests of the structure and mechanical properties, it was found that in the case of walls of AAA preparations the determined mechanical and structural parameters varied widely and were statistically significantly different from the results obtained for NAAs. Therefore, an analysis was conducted of the effect of the number of elastic lamellae and collagen fibres on the parameters determined during the examination of mechanical properties. The results of the analysis are collected and presented in Table 7 .

Table 7. Pearson's correlation coefficients $(R)$ between the determined mechanical parameters and the number of elastic lamellae and collagen fibres for specimens excised from AAA walls in the circumferential and longitudinal directions

\begin{tabular}{|l|c|c|c|c|}
\cline { 2 - 5 } \multicolumn{1}{c|}{} & \multicolumn{2}{c|}{ AAAc } & \multicolumn{2}{c|}{ AAAa } \\
\cline { 2 - 5 } \multicolumn{1}{c|}{} & $N_{E}$ & $N_{C}$ & $N_{E}$ & $N_{C}$ \\
\hline$\sigma_{M}[\mathrm{MPa}]$ & 0.32 & 0.86 & 0.22 & 0.71 \\
\hline$M T M[\mathrm{MPa}]$ & 0.09 & 0.42 & 0.07 & 0.44 \\
\hline$\lambda_{I P}[-]$ & 0.23 & 0.37 & 0.19 & 0.32 \\
\hline$\sigma_{I P}[\mathrm{MPa}]$ & 0.29 & 0.33 & 0.16 & 0.46 \\
\hline$M_{T} M_{L}[\mathrm{MPa}]$ & 0.83 & 0.25 & 0.42 & 0.07 \\
\hline$M T M_{H}[\mathrm{MPa}]$ & 0.30 & 0.84 & 0.31 & 0.53 \\
\hline
\end{tabular}

Anisotrophy and nonlinearity index of AAAs

\begin{tabular}{|l|c|c|}
\hline & $N_{E}$ & $N_{C}$ \\
\hline$A[-]$ & 0.38 & 0.47 \\
\hline $\operatorname{det} \boldsymbol{F}[-]$ & 0.41 & 0.49 \\
\hline \multicolumn{2}{|c|}{ Constitutive parameters of AAAs } \\
\hline
\end{tabular}

Constitutive parameters of AAAs

\begin{tabular}{|l|c|c|}
\hline & $N_{E}$ & $N_{C}$ \\
\hline$\mu[\mathrm{MPa}]$ & 0.87 & 0.32 \\
\hline$k_{1}[\mathrm{MPa}]$ & 0.21 & 0.69 \\
\hline$k_{2}[-]$ & 0.34 & 0.56 \\
\hline$\alpha\left[^{\circ}\right]$ & 0.13 & 0.28 \\
\hline
\end{tabular}

The values of Pearson's correlation coefficient $(R)$ indicated a significant differentiation of the relationship between individual mechanical and constitutive parameters, and the number of elastic lamellae and collagen fibres, ranging from very weak, through mod- erate and good, to very strong. For most of the analysed correlations, the correlation coefficients were higher for specimens excised in the circumferential than axial direction.

\section{Discussion}

This study characterized the mechanical and structural properties of human AAAs, harvested intraoperatively, and compared them to NAAs, harvested post-mortem from subjects of the corresponding age and sex. The structural composition was examined by histological techniques and supported by SEM imaging. The mechanical properties were determined using uniaxial tensile tests for two main directions: circumferential and axial. The results showed significant differences in the behaviour of NAAs and AAAs with respect to structural composition.

The walls of AAAs were characterised by a significantly higher stiffness (defined here as MTM) compared to the walls of normal aortae, irrespective of the testing direction, and lower strength, which was significantly lower in the circumferential direction. Similar results were also obtained by other researchers in both uniaxial tension tests [24], [27], [28] and in biaxial tests [7]. Tensile strength strongly correlated with the number of collagen fibres, which is a proof that collagen degradation is the main cause of wall damage, i.e., aneurysm rupture [25]. A typical nonlinear strength curve (Fig. 7) had a different characteristic in terms of low- and high-stretch domains. This results from the participation of elastic lamellae and collagen fibres in the load transfer process, which is typical of soft tissues rich in collagen [14]-[16], [31]. The above phenomenon has been well described [12]; the operational ranges of both structures have been defined using techniques of selective digestion of load-bearing components from aortic walls, separately elastic lamellae and collagen fibres. Elastic lamellae are responsible for the behaviour of the blood vessel wall in the low deformation range, while collagen fibres are responsible for the mechanical response of the vessel wall in the high deformation range. A typical characteristic curve, i.e., the toe region, results from gradual involvement of collagen fibres into the process of transfer of mechanical loads [8]. The dimensionless parameter of the model $\left(k_{2}\right)$ reflects the degree of non-linearity caused by gradual involvement of collagen fibres in the process of transfer of mechanical loads. The value of this parameter, which at a good level correlated with the number of collagen 
fibres, was significantly lower for AAAs, which means that strength curves for AAA walls were less nonlinear than NAA walls (Fig. 7). An assessment of the strength characteristic curve allowed to determine the IP describing the change of the curve slope. Following Niestrawska et al. [21], the term "inflections point" is understood as the point of the stress-stretch curve where collagen takes over the mechanical response and the material rapidly stiffens. The IP coordinates $\left(\lambda_{I P}, \sigma_{I P}\right)$ for AAAs were significantly lower in the circumferential direction and slightly lower in the axial direction, compared to NAAs. The IP coordinates were relatively weakly correlated with the number of elastic lamellae and moderately correlated with the number of collagen fibres. After passing through the IPs, the curves of AAAs had a steeper slope than in the case of NAAs, and the toe regions were narrower (Fig. 7). The division of the curve into two ranges determined by the IP enabled the assessment of the material stiffness in conjunction with the scope of work of individual load-bearing structures [12], elastic lamellae in the low-stretch domains, and collagen fibres in the high-stretch domains. AAAs, when compared to NAAs, were characterised by insignificantly lower elastic modulus for the low-stretch domains $\left(M T M_{L}\right)$ and significantly higher elastic modulus in the circumferential direction for the high-stretch domains $\left(M T M_{H}\right)$. A similar relationship was observed for the stress-like parameters of the constitutive model $\mu$ and $k_{1}$. They are associated with stiffness of the isotropic ground matrix and collagen fibres, respectively. The $\mu$ was significantly higher for NAAs, and the $k_{1}$ parameter was significantly higher for AAAs. The correlation of these parameters with the number of collagen fibres $\left(N_{C}\right)$ and elastic lamellae $\left(N_{E}\right)$ (Table 7) showed strong relationships for the pairs: $M T M_{L}-N_{E}$ and $\mu-N_{E}$, and also $M T M_{H}-N_{C}$ and $k_{1}-N_{C}$.

The angle between the circumferential direction and the main directions of the fibres $(\alpha)$ varied widely for AAAs (Table 3), which indicates its high variability in the case of aneurysms. This means that the arrangement of collagen fibres is less ordered than in the case of NAAs. In the case of aneurysmal walls, anisotropy was clearly less marked, which was consistent with a previous report [13], and aneurysms were very slightly more compliant circumferentially than axially (Table 5). The anisotropy index for NAAs was in general agreement with the data observed for older aortae (over 50 years old) [11]; NAAs demonstrated higher circumferential compliance. Both NAAs and AAAs were incompressible materials (Table 5), which is consistent with the widely accepted assumption proved by Carew at al. [2] already in 1968 .
Despite the fact that the performed correlation analyses accurately reflect the dependence of individual mechanical parameters on the contents of load-bearing structural components, this dependence is not exhaustive. Assuming that, unlike in the remodelling of arterial walls during the development of atherosclerosis [14], [17], no atypical formations appear in the structure of AAAs as a result of remodelling, there are at least several properties of the fibrotic extracellular matrix that can significantly affect the mechanical properties and parameters of material models. These include, among others, the degree of collagen crosslinking, collagen turn-over, degree of collagen packing, fragmentation of elastic lamellae, degree of hydration (proteoglycan content), and spatial fibre arrangement, described here by the angle between the circumferential direction and the main directions of the fibres $(\alpha)$.

In all analysed cases, correlation coefficients were higher for specimens excised in the circumferential direction. This result is justified due to the specific structure of the blood vessel walls. In addition, AAAs always grow in the circumferential direction relative to the long axis of the vessel and this direction also shows the most characteristic changes in the mechanical properties during progression of AAAs' development. The complex architecture of arterial tissue is represented in constitutive modelling as a fibre-reinforced composite [9], [21], in which two families of collagen fibres are embedded in an isotropic ground matrix. In general, the material parameters obtained in this study are consistent with the results presented by other authors for the same model and models extended by a larger number of structural parameters, including fibre dispersion in the in-plane and out-plane directions [21], [22].

All the analysed mechanical parameters are characterised by significant variability, which may be conditioned by many factors, particularly individual variability, since each of the specimens may have a different structure. On the other hand, diversity in structural composition of AAAs may be determined by, among others, the presence of mural thrombus [28], geometry of the aneurysm (in particular wall thickness) [24], and development of comorbidities, including atherosclerotic plaques [15] and dissection [16]. Regardless of the factors affecting tissue remodelling, the structural composition of AAAs depends on the stage of the disease. The composition of abdominal walls during aneurysm development showed a significant reduction in the concentration of elastic lamellae (approx. 85\%) and collagen fibres (approx. 20\%) (Table 3). The loss of elastic lamellae in the aneurysmal wall can range from $63 \%$ to $92 \%$ [30]. While the content of 
elastin fibres in the aneurysmal wall always decreases, the collagen content may increase [4], remain unchanged, or decrease [20], similar to the results of tests performed in this study. The character of structural changes occurring in the wall of the abdominal aorta in the process of aneurysm growth has been systematized. Thompson and Baxter [26] proposed a threestage identification model of AAA growth based on the characteristic structural changes and the size of the aneurysm. The first phenomenon occurring in the process of aneurysm development is the fragmentation of elastic lamellae and their reduced concentration in the medial layer of the aortic wall, which is also confirmed by the results presented here. Degradation of elastin fibres in the media of the aortic wall reduces the ability of the vessel to transfer tensile stresses and induces compensatory collagen production. The increase in collagen content in the aneurysmal wall suggests the existence of repair or compensatory processes. In the second stage, collagen degradation and synthesis proceed with similar intensity. However, in the third stage of AAA development, an imbalance occurs between collagen degradation and its synthesis, which causes a decrease in the tensile strength of the aortic walls, and under hypertensive conditions this leads to aneurysm rupture [25]. Tensile strength in the circumferential direction in the case of ruptured AAAs is $30-40 \%$ lower compared to the specimens excised from aneurysms during elective surgeries [3].

Tests of the mechanical properties of the NAA and AAA walls were performed under uniaxial tensile loading in two perpendicular directions: circumferential and axial. This type of test was treated as equivalent to biaxial tests, which are considered to be best at mimicking the physiological conditions [9], [21]. The test conditions were chosen to balance the need for restoration of physiological conditions against the technical capabilities of the mechanical tests. Suitable conditions representative of the in vivo physiological environment for the vascular tissues are as follows: hydration and ambient temperature equal to $37^{\circ} \mathrm{C}$, stress distribution without artificial local concentration, and strain rate mimicking the normal cardiac systole [29]. In this study, mechanical tests were conducted at a much lower speed to avoid viscoelastic effects [23] resulting from the action of smooth muscle cells. Smooth muscle cells are responsible for active behaviour of arterial tissues under mechanical loads conditions. Because the purpose of the study was to assess passive mechanical properties of arterial tissues, the potential impact of active mechanical response of the tissues should be minimized so that the impact of this component on the final measurement result is negligible. During the tests, the tissues were kept hydrated but due to the use of a non-contact deformation measuring device - a video extensometer, no tests were performed in a water bath at $37{ }^{\circ} \mathrm{C}$, which may induce stiffening of arterial tissue [5]. The local stress concentration within the specimen material was eliminated by maintaining the width to length (WL) ratio below 1:1 that was found to be most suited for tensile testing [29].

\section{Conclusions}

This study assessed the effect of pathological remodelling on the mechanical behaviour of AAAs. Structural heterogeneity of the specimens and severity of aneurysmal degeneration were quantified by counting collagen fibres and elastic lamellae. Using a large, heterogeneous group of AAA specimens, it was demonstrated that the number of collagen fibres and elastic lamellae is well correlated with the mechanical properties determined under uniaxial loadings and constitutive parameters determined for the commonly used constitutive model [9]. Hence, aneurysm development with the concomitant changes in mechanical properties can be assessed over time based on the number of loadbearing structural elements. In particular, the number of collagen fibres has a key clinical role and potential for AAA rupture evaluation because the coefficient of correlation with failure strength was observed at the highest level. As a next step, a mechanobiological model of AAA development should be developed.

\section{Limitations}

The representative control group was selected on the basis of the age structure and sex distribution in the experimental aneurysmatic group. In the aortic normal walls classified as the control group showed atherosclerotic changes with different pathologies, which is typical in this age range [14]. Thus, the determined mechanical properties of NAA are affected by pathological arteriosclerotic remodelling.

Tests were carried out on specimens excised only from the anterior walls of AAAs during elective repair procedures. The sampling conditions, in particular the overriding need to ensure the welfare of the patient/ donor, prevented an analysis of the mechanical properties of AAA walls that would consider other locations, e.g., lateral or posterior walls. While examining 
the effect of the sampling site on the mechanical conditions, Thubrikar et al. [27] noted that stiffness and strength were highest for lateral AAA surfaces and strength was lowest for anterior AAA surfaces.

The applied constitutive model contains five parameters, including one (angle between the circumferential direction and the main directions of the fibres, $\alpha$ ) describing structural properties of the tested material. This study did not analyse the arrangement and pattern of collagen fibres in the wall. It is known that for NAA this angle averages 45 . In the case of AAA, on the other hand, the value of $\alpha$ varies widely depending on the degree of tissue remodelling. Structural imaging methods, such as SEM and histology, are not recommended methods for assessing the spatial distribution of collagen fibres in tissues. Volumetric high-resolution imaging methods are preferable, especially second-harmonic generation imaging [21], [22]. In this study, all parameters of the model, including the angle describing fibers arrangement, were determined by fitting the model to experimental data.

\section{Acknowledgements}

This study was carried out as part of a project financed by the National Science Centre (decision no. DEC-2013/09/D/ST8/04007).

I would like to thank the following people for helping me to finalize this project: Krzysztof Maksymowicz and Jan Gnus for collecting tissue material for testing, Katarzyna Kaleta-Kuratewicz for performing histological staining, and Justyna Niestrawska for background to constitutive modelling.

\section{References}

[1] AleXAnder J., The pathobiology of aortic aneurysms, J. Surg. Res., 2004, 117, 163-175.

[2] Carew T., Vaishnav R., Patel D., Compressibility of the arterial wall, Circ. Res., 1968, 23, 61-68.

[3] Dimartino E., Bohra A., Geest J., Gupta N., Makaroun M., VORP D., Biomechanical properties of ruptured versus electively repaired abdominal aortic aneurysm wall tissue, J. Vasc. Surg., 2006, 43, 570-676.

[4] Eugster T., Huber A., Obeid T., Schwegler I., Gurke L., STIERLI P., Aminoterminal propeptide of type III procollagen and matrix metalloproteinases-2 and -9 failed to serve as serum markers for abdominal aortic aneurysm, Eur. J. Vasc. Endovasc., 2005, 29, 378-382.

[5] FunG Y.C.B., Elasticity of soft tissue in simple elongation, Am. J. Physiol., 1976, 213, 1532-1534.

[6] Gąsior-GŁogowska M., Komorowska M., Hanuza J., PTAK M., Kobielarz M., Structural alteration of collagen $\mathrm{fi}$ bres - spectroscopic and mechanical studies, Acta Bioeng. Biomech., 2010, 12, 55-62.

[7] GeEST J., SACKS M., VORP D., The effects of aneurysm on the biaxial mechanical behavior of human abdominal aorta, J. Biomech., 2006, 39, 1324-1334.
[8] Hanuza J., MączKa M., GąSior-GŁogowska M., Komorowska M., Kobielarz M., BĘDZiŃSKi R., Szotek S., MaKsymowicz K., Hermanowicz K., FT-Raman spectroscopic study of thoracic aortic wall subjected to uniaxial stress, J. Raman. Spectrosc., 2010, 41, 1163-1169.

[9] Holzapfel G., Gasser T., OGden R., A new constitutive framework for arterial wall mechanics and a comparative study of material models, J. Elasticity, 2000, 61, 1-48.

[10] JOHnSton K.W., Rutherford R.B., TiLSON M.D., SHAH D.M., Hollier L., Stanley J.C., Suggested standards for reporting on arterial aneurysms, J. Vasc. Surg., 1991, 13 (3), 452-458.

[11] Kamenskiy A.V., Dzenis Y.A., Kazmi S.A.J., Pemberton M.A., Pipinos I.I.I., Phillips N.Y., Herber K., WoOdFord T., Bowen R.E., Lomneth C.S., MaC-Taggart J.N., Biaxial mechanical properties of the human thoracic and abdominal aorta, common carotid, subclavian, renal and common iliac arteries, Biomech. Model Mechanobiol., 2014, 13, 1341-1359.

[12] Kobielarz M., ChwiŁKowska A., TureK A., Maksymowicz K., MARCINIAK M., Influence of selective digestion of elastin and collagen on mechanical properties of human aortas, Acta Bioeng. Biomech., 2015, 17, 55-62.

[13] KOBIELARZ M., JANKOWSKI L., Experimental characterization of the mechanical properties of the abdominal aortic aneurysm wall under uniaxial tension, J. Theor. Appl. Mech., 2013, 51(4), 949-958.

[14] Kobielarz M., Kozuń M., Gąsior-GŁogowska M., CHWIŁKOWSKA A., Mechanical and structural properties of different types of human aortic atherosclerotic plaques, J. Mech. Behav. Biomed. Mater, 2020, 109, DOI: 10.1016/ j.jmbbm.2020.103837.

[15] Kobielarz M., Kozuń M., Kuzan A., Maksymowicz K., Witkiewicz W., Pezowicz C., The intima with early atherosclerotic lesions is load-bearing component of human thoracic aorta, Biocybern. Biomed. Eng., 2017, 37, 35-43.

[16] Kozuń M., Kobielarz M., Chwilkowska A., Pezowicz C., The impact of development of atherosclerosis on delamination resistance of the thoracic aortic wall, J. Mech. Behav. Biomed. Mater, 2018, 79, 292-300.

[17] Kuzan A., ChwiŁkowska A., Pezowicz C., Witkiewicz W., Gamian A., MaKsymowicz K., Kobielarz M., The content of collagen type II in human arteries is correlated with the stage of atherosclerosis and calcification foci, Cardiovasc. Pathol., 2017, 28, 21-27.

[18] Lederle F.A., Kyriakides T.C., Stroupe K.T., Freischlag J.A., Padberg F.T., Matsumura J.S., Huo Z., Johnson G.R., Open versus endovascular repair of abdominal aortic aneurysm, N. Engl. J. Med., 2019, 380, 2126-2135.

[19] Maksymowicz K., Kobielarz M., Czogala J., Potential indicators of the degree of abdominal aortic aneurysm development in rupture risk estimation, Adv. Clin. Exp. Med., 2011, 20 (2), 221-225.

[20] McGee G., Baxter T., Shively V., Chisholm R., McCarthy W., Flinn W., Yao J., Pearce W., Aneurysm or occlusive disease - factors determining the clinical course of atherosclerosis of the infrarenal aorta, Surgery, 1991, 110, 370-375.

[21] Niestrawska J.A., Regitnig P., Viertler C., Cohnert T.U., BABU A.R., Holzapfel G.A., The role of tissue remodeling in mechanics and pathogenesis of abdominal aortic aneurysms, Acta Biomater., 2019, 88, 149-161.

[22] Niestrawska J.A., Viertler C., Regitnig P., Cohnert T.U., SOMmer G., Holzapfel G.A., Microstructure and me- 
chanics of healthy and aneurysmatic abdominal aortas: experimental analysis and modeling, J. R. Soc. Interface, 2016, 13, 20160620.

[23] Pierce D., Maier F., Weisbecker H., Viertler C., Verbrugghe P., Famaey N., Fourneau I., Herijgers P., Holzapfel G.A., Human thoracic and abdominal aortic aneurysmal tissues: Damage experiments, statistical analysis and constitutive modeling, J. Mech. Behav. Biomed. Mater, 2015, 41, 92-107.

[24] Raghavan M., Kratzberg J., Tolosa E., Hanaoka M., WALKER P., DASILVA E., Regional distribution of wall thickness and failure properties of human abdominal aortic aneurysm, J. Biomech., 2006, 39, 3010-3016.

[25] Sakalihasan N., Limet R., Defawe O., Abdominal aortic aneurysm, Lancet, 2005, 365, 1577-1589.

[26] THOMPSON R., BAXTER T., MMP Inhibition in abdominal aortic aneurysms rationale for a prospective randomized clinical trial, Ann. NY. Acad. Sci., 1999, 878, 159-178.
[27] Thubrikar M., Labrosse M., Robicsek F., Al-Soudi J., FOWLER B., Mechanical properties of abdominal aortic aneurysm wall, J. Med. Eng. Technol., 2001, 25, 133-142.

[28] Vorp D., Lee P., Wang D., Makaroun M., Ogawa S., WeBSTER M., Association of intraluminal thrombus in $a b$ dominal aortic aneurysm with local hypoxia and wall weakening, J. Vasc. Surg., 2001, 34, 291-299.

[29] Walsh M.T., CunNane E.M., Mulvihill J.J., AKYILDIZ A.C., GiJSEN F.J.H., HolzAPfEl G.A., Uniaxial tensile testing approaches for characterisation of atherosclerotic plaques, Journal of Biomechanics, 2014, 47, 793-804.

[30] Watton P., Hill N., Heil M., A mathematical model for the growth of the abdominal aortic aneurysm, Biomech. Model Mechanobiol., 2004, 3, 98-113.

[31] Wysocki M., Kobus K., Szotek S., Kobielarz M., KUROPKA P., BĘDZIŃSKI R., Biomechanical effect of rapid mucoperiosteal palatal tissue expansion with the use of osmotic expanders, J. Biomech., 2011, 44, 1313-1320. 